\title{
Non-HDL-C Is More Stable Than LDL-C in Assessing the Percent Attainment of Non-fasting Lipid for Coronary Heart Disease Patients
}

\author{
Li-Ling Guo 1,2,3,4, Yan-qiao Chen 1,2,3,4, Qiu-zhen Lin 1,2,3,4, Feng Tian 1,2,3,4, \\ Qun-Yan Xiang ${ }^{1,2,3,4}$, Li-yuan Zhu ${ }^{1,2,3,4}$, Jin Xu ${ }^{1,2,3,4}$, Tie Wen ${ }^{5}$ and Ling Liu ${ }^{1,2,3,4 *}$ \\ ${ }^{1}$ Department of Cardiovascular Medicine, The Second Xiangya Hospital, Central South University, Changsha, China, \\ ${ }^{2}$ Research Institute of Blood Lipid and Atherosclerosis, Center South University, Changsha, China, ${ }^{3}$ Modern Cardiovascular \\ Disease Clinical Technology Research Center of Hunan Province, Changsha, China, ${ }^{4}$ Cardiovascular Disease Research \\ Center of Hunan Province, Changsha, China, ${ }^{5}$ Department of Emergency, The Second Xiangya Hospital, Central South \\ University, Changsha, China
}

OPEN ACCESS

Edited by:

Zhong Wang,

University of Michigan, United States

Reviewed by:

Tetsuro Miyazaki,

Juntendo University Urayasu

Hospital, Japan

Alon Schaffer,

Azienda Ospedaliero Universitaria

Maggiore della Carità, Italy

${ }^{*}$ Correspondence:

Ling Liu

feliuling@csu.edu.cn

Specialty section:

This article was submitted to

Cardiovascular Metabolism,

a section of the journal

Frontiers in Cardiovascular Medicine

Received: 04 January 2021 Accepted: 08 March 2021

Published: 01 April 2021

Citation:

Guo L-L, Chen Y-q, Lin Q-z, Tian F, Xiang Q-Y, Zhu L-y, Xu J, Wen T and Liu L (2021) Non-HDL-C Is More Stable Than LDL-C in Assessing the Percent Attainment of Non-fasting Lipid for Coronary Heart Disease

Patients.

Front. Cardiovasc. Med. 8:649181.

doi: 10.3389/fcvm.2021.649181
This study aimed to compare the percentage attainment of fasting and non-fasting LDL-C and non-HDL-C target levels in coronary heart disease (CHD) patients receiving short-term statin therapy. This study enrolled 397 inpatients with CHD. Of these, 197 patients took statins for $<1$ month $(\mathrm{m})$ or did not take any statin before admission (CHD1 group), while 204 patients took statins for $\geq 1 \mathrm{~m}$ before admission (CHD2 group). Blood lipid levels were measured at 0,2 , and $4 \mathrm{~h}$ after a daily breakfast. Non-fasting LDL-C and non-HDL-C levels significantly decreased after a daily meal $(P<0.05)$. Both fasting and non-fasting LDL-C or non-HDL-C levels were significantly lower in the CHD2 group. The percentage attainment of LDL-C $<1.4 \mathrm{mmol} / \mathrm{L}$ at 2 and $4 \mathrm{~h}$ after a daily breakfast was significantly higher than that during fasting $(P<0.05)$, but the percent attainment of non-fasting non-HDL-C $<2.2 \mathrm{mmol} / \mathrm{L}$ was close to its fasting value $(P>0.05)$. Analysis of c-statistic showed that non-fasting cut-off points for LDL-C and non-HDL-C were 1.19 and $2.11 \mathrm{mmol} / \mathrm{L}$, corresponding to their fasting goal levels of 1.4 and 2.2 $\mathrm{mmol} / \mathrm{L}$, respectively. When post-prandial LDL-C and non-HDL-C goal attainments were re-evaluated using non-fasting cut-off points, there were no significant differences in percentage attainment between fasting and non-fasting states. Non-HDL-C is more stable than LDL-C in assessing the percent attainment of non-fasting lipid for coronary heart disease patients. If we want to use LDL-C to assess the percent attainment of post-prandial blood lipids, we may need to determine a lower non-fasting cut-off point.

Keywords: coronary heart disease, non-fasting, low-density lipoprotein cholesterol, non-high-density lipoprotein cholesterol, cut-off points

\section{INTRODUCTION}

Elevated cholesterol level is an independent risk factor for coronary heart disease (CHD). To reduce the risk of ischemic events for CHD patients, fasting level of low-density lipoprotein cholesterol (LDL-C) should be controlled to $<1.4 \mathrm{mmol} / \mathrm{L}$ as the primary target, then that of non-high-density lipoprotein cholesterol (non-HDL-C) should be $<2.2 \mathrm{mmol} / \mathrm{L}$ as the secondary target of cholesterol control according to the 2019 European guidelines (1). 
It is increasingly believed that atherosclerosis is a postprandial phenomenon because, at least with respect to lipids, we are in the post-prandial phase for the most part of the day (2). Considering that either fasting or non-fasting (i.e., post-prandial) LDL-C level has a similar predictive value for all-cause death and cardiovascular death $(3,4)$, non-fasting lipids detection at a random time-point within at least $8 \mathrm{~h}$ after a daily meal has been recommended in the primary and secondary prevention against CHD (5-8). However, both LDL-C and non-HDL-C levels show a tendency of decrease in the non-fasting state (9-11). Moreover, there are only fasting cholesterol-lowering targets but not non-fasting ones in the published guidelines (1, 12-15). It is uncertain whether these fasting targets are applicable to assessing cholesterol control as well as how to evaluate it in the non-fasting stage.

Recently, we observed more substantial reductions in LDL-C and non-HDL-C levels in Chinese subjects with CHD at 2 and $4 \mathrm{~h}$ after a daily breakfast $(9,16)$, appearing to be greater than those reported in large-scale clinical studies conducted in other countries $(10,11,17,18)$, although the potential cause remains uncertain. Additionally, it was proposed that non-HDL-C level may be a better prognostic factor than LDL-C level to evaluate the risk of future cardiovascular events (19-24). Furthermore, non-fasting fluctuation of non-HDL-C level seem to be smaller than that of LDL-C (24). Nevertheless, there have been no studies comparing the goal attainment of LDL-C with that of non-HDL$\mathrm{C}$ in the non-fasting state.

Therefore, this study aimed to compare the percent attainments of fasting and non-fasting LDL-C and nonHDL-C reaching their fasting targets in CHD patients receiving short-term statins therapy. Furthermore, analysis of c-statistic or receiver operating characteristic curve (ROC) analysis was used to determine the non-fasting cut-off points corresponding to their fasting targets, and the percent attainments of non-fasting LDL-C and non-HDL-C were re-evaluated according to the non-fasting cut-off points.

\section{MATERIALS AND METHODS}

\section{Study Population}

From March 2017 to July 2019, 397 inpatients with CHD were enrolled from the Department of Cardiovascular Medicine of the Second Xiangya Hospital, Central South University. A total of 84 patients did not take any statins and 109 patients took statins for $<1$ month (m) before admission (CHD1 group), and 204 patients took statins for $\geq 1 \mathrm{~m}$ before admission (CHD2 group). The definition of CHD was coronary atherosclerosis confirmed by coronary angiography and/or a history of myocardial infarction in patients with angina pectoris (AP). We asked all patients about their medical history and use of medication before enrollment. Patients with autoimmune disease, hepatic disorders, renal disease, cancer, or other serious diseases were excluded. The study was approved by the Ethics Committee of the Second Xiangya Hospital of Central South University. Informed consent was obtained from all patients.

\section{Specimen Collection}

All enrolled participants ate breakfast between 7 a.m. and 8 a.m. according to their regular diets after overnight fast for at least $8 \mathrm{~h}$. The patients in this study are all Han nationality, and their daily breakfast were rich in carbohydrate, including steamed bread, noodles, rice porridge, and so on, which were purchased from the cafeteria in our hospital or brought from home according to their dietary habits. During 4-h test, subjects were allowed only to drink a little water and walk slowly. Drinking wine or eating any food were not recommended.

\section{Determination of Blood Lipids Levels}

Serum total cholesterol (TC) and triglyceride (TG) levels were measured using automated enzymatic assays. Serum highdensity lipoprotein cholesterol (HDL-C) and LDL-C levels were measured using a chemical masking method (25). All measurements, including that of albumin, were carried out on a fully automatic biochemical analyser (Hitachi 7170A, Hitachi Inc., Tokyo, Japan) and performed by the expert who didn't know the details of the research (26). Non-HDL-C equals to TC minus HDL-C.

\section{Statistical Analysis}

Data were analyzed using SPSS version 19.0. (IBM Corp., Armonk, NY, USA) and Prism 6.0 (GraphPad Inc., San Diego, CA, USA). Continuous variable values data were shown as mean \pm standard deviation (SD), and categorical data were shown as numbers and percentages. The $t$-test and chi-square test were used to analyse continuous variables and categorical variables, respectively. The optimal cut-off points for fasting LDL-C (1.4 $\mathrm{mmol} / \mathrm{L})$ and non-HDL-C (2.2 $\mathrm{mmol} / \mathrm{L})$ were determined using receiver operating characteristic (ROC curve) analysis. Based on the ROC curve, values determined using Youden analysis were used as cut-off points. All tests were two-tailed, and $P<0.05$ was considered statistically significant.

\section{RESULTS}

\section{Clinical Characteristics and Fasting Blood Lipids in Two CHD Groups}

The baseline characteristics of the CHD patients are shown in Table 1. Both groups were similar in terms of age, sex, body mass index, percentages of hypertension, current smoking, and diabetes mellitus. There were $56.5 \%$ patients taking statins $<1 \mathrm{~m}$ and $43.5 \%$ patients without statins treatment before admission in CHD1 group. Fasting serum levels of TC, LDL-C and nonHDL-C in CHD2 group were significantly lower than those in CHD1 group $(P<0.05)$. The differences in fasting serum TG and HDL-C levels between the groups did not differ significantly (Table 1). The proportion of STEMI and NSTEMI patients in CHD2 group was less than that in CHD1 group $(P<0.05)$. However, the proportion of schemic cardiomyopathy in CHD2 group was more than that in CHD1 group $(P<0.05)$. The differences in vascular disease (single or multiple vessel disease) did not differ significantly (Table 1). 
TABLE 1 | Baseline characteristics of the study population.

\begin{tabular}{|c|c|c|}
\hline & CHD1 $(n=193)$ & CHD2 $(n=204)$ \\
\hline Age $(y, S D)$ & $60.3 \pm 9.3$ & $62.0 \pm 8.7$ \\
\hline Men, $n(\%)$ & $156(80.8)$ & $157(77.0)$ \\
\hline BMI $\left(\mathrm{kg} / \mathrm{m}^{2}, \mathrm{SD}\right)$ & $24.5 \pm 3.5$ & $24.9 \pm 3.0$ \\
\hline Hypertension, $n(\%)$ & $141(73.1)$ & $152(74.5)$ \\
\hline Current smoking, $n$ (\%) & $110(57.0)$ & $103(50.5)$ \\
\hline $\mathrm{DM}, n(\%)$ & $51(26.4)$ & $67(32.8)$ \\
\hline \multicolumn{3}{|l|}{ Taking statins, $n$ (\%) } \\
\hline Statins $\geq 1 \mathrm{~m}$ & 0 & $204(100)^{\star}$ \\
\hline Statins $<1 \mathrm{~m}$ & $109(56.5)$ & $0^{*}$ \\
\hline No statins & $84(43.5)$ & $0^{*}$ \\
\hline \multicolumn{3}{|l|}{ Vascular disease, $n(\%)$} \\
\hline Single vessel disease & $43(22.3)$ & $36(17.6)$ \\
\hline Multiple vessel disease & $132(68.4)$ & $146(71.6)$ \\
\hline Without CAG & $18(9.3)$ & $22(10.8)$ \\
\hline \multicolumn{3}{|l|}{ CHD subtype, $n(\%)$} \\
\hline STEMI & $14(7.3)$ & $4(2.0)^{\star}$ \\
\hline NSTEMI & $32(16.6)$ & $13(6.4)^{\star}$ \\
\hline UA & $102(52.8)$ & $114(55.9)$ \\
\hline SAP & $31(16.1)$ & 42 (20.6) \\
\hline Schemic cardiomyopathy & $4(2.1)$ & $16(7.8)^{\star}$ \\
\hline Others & $10(5.2)$ & $15(7.4)$ \\
\hline TC (mmol/L, SD) & $4.32 \pm 0.91$ & $3.93 \pm 1.03^{*}$ \\
\hline LDL-C (mmol/L, SD) & $2.72 \pm 0.79$ & $2.43 \pm 0.90^{*}$ \\
\hline Non-HDL-C (mmol/L, SD) & $3.20 \pm 0.96$ & $2.92 \pm 0.98^{*}$ \\
\hline HDL-C (mmol/L, SD) & $1.12 \pm 0.27$ & $1.01 \pm 0.25$ \\
\hline TG (mmol/L, SD) & $1.74 \pm 1.11$ & $1.84 \pm 1.29$ \\
\hline
\end{tabular}

CHD1 group: CHD patients taking statins $<1 \mathrm{~m}$ and without statins treatment before admission. CHD2 group: CHD patients taking statins $\geq 1 \mathrm{~m}$ before admission. BMI, body mass index; DM, diabetes mellitus; SAP, stable angina pectoris; Others in CHD subtype: Including coronary microangiopathy, etc. TG, triglyceride; TC, total cholesterol; HDL-C, high-density lipoprotein cholesterol; LDL-C, low-density lipoprotein. Continuous variable values were reported as mean $\pm S D$, and categorical data were reported as numbers and percentages. ${ }^{*} P<0.05$ when compared with $C H D 1$ group.

According to the time of statin using before admission, all patients were divided into three groups: CHD1 group (nonstatin taking, $n=93$ ), CHD2 group [short-term statin taking ( $<1 \mathrm{~m}), n=109$ ] and CHD3 group [long-term statin taking ( $\geq 1 \mathrm{~m}), n=204]$, which were added as the supplement data in the new manuscript due to the small sample size in this study (Supplementary Table 1).

\section{Comparison of Changes in Non-fasting Blood Lipids in the Two Groups}

Levels of LDL-C and non-HDL-C decreased significantly at 2 and $4 \mathrm{~h}$ after a daily breakfast $(P<0.05)$. Non-fasting LDL-C and non-HDL-C in CHD2 group were significantly lower than those in CHD1 group $(P<0.05$; Figures 1A,B). When the data at 2 and $4 \mathrm{~h}$ after a daily meal as a whole were used as non-fasting data for further analysis, nonfasting reductions in LDL-C were 0.47 and $0.46 \mathrm{mmol} / \mathrm{L}$, and non-HDL-C were 0.26 and $0.24 \mathrm{mmol} / \mathrm{L}$ in $\mathrm{CHD} 1$ and
CHD2 groups, respectively (Figure 1C). The percentages of reduction in LDL-C were 17.1 and $18.5 \%$, and 7.2 and $7.7 \%$ in non-HDL-C in CHD1 and CHD2 groups, respectively (Figure 1D). There were no significant differences in the absolute reduction or percentage of reduction in LDL-C or non-HDL-C level between the groups. However, nonfasting reductions in LDL-C were greater than those of non-HDL-C (Figure 1B).

Non-fasting albumin levels were measured in 89 patients among all CHD patients. There was no significant change in the albumin level after a daily breakfast (Table 2 ), whereas the postprandial TC, HDL-C, LDL-C, and non-HDL-C level dropped significantly $(P<0.05$; Table 2$)$.

\section{Evaluating Goal Attainments of LDL-C and Non-HDL-C According to Various Targets in CHD2 Group}

According to the 2019 European guidelines (1), the percent attainment of LDL-C $<1.4 \mathrm{mmol} / \mathrm{L}$ in the fasting state was significantly lower than that of non-HDL-C $<2.2 \mathrm{mmol} / \mathrm{L}(10.8$ vs. $32.8 \%, P<0.001)$. After a daily breakfast, the percent attainment of non-HDL-C and LDL-C gradually tightened, and there was statistical difference only at $2 \mathrm{~h}$. The percent attainment of LDL-C at 2 or $4 \mathrm{~h}$ was significantly higher than its fasting value $(P<0.05)$, but there is no difference in non-HDL-C between fasting and post-prandial values $(P>0.05$; Figure 2A).

ROC curve analysis showed that non-fasting cut-off points for LDL-C and non-HDL-C at $4 \mathrm{~h}$ were $1.19 \mathrm{mmol} / \mathrm{L}$ (sensitivity $90.1 \%$, specificity $77.3 \%$, and AUC 0.904 ) and 2.11 $\mathrm{mmol} / \mathrm{L}$ (sensitivity $87.6 \%$, specificity $80.6 \%$, and AUC 0.913 ), corresponding to their fasting goal levels of 1.4 and $2.2 \mathrm{mmol} / \mathrm{L}$, respectively (Figures $\mathbf{2 B}, \mathbf{C}$ ).

According to the non-fasting cut-off points, the percent attainment of LDL-C $<1.19 \mathrm{mmol} / \mathrm{L}$ at 2 or $4 \mathrm{~h}$ was 14.7 or $17.2 \%$, which was close to the percent attainment of LDL-C $<1.4 \mathrm{mmol} / \mathrm{L}$ in the fasting state. The percent attainment of non-HDL-C $<2.11 \mathrm{mmol} / \mathrm{L}$ at 2 or $4 \mathrm{~h}$ was close to the percent attainment of non-HDL-C $<2.2 \mathrm{mmol} / \mathrm{L}$ in the fasting state. Moreover, the percent attainment of LDL-C $<1.19 \mathrm{mmol} / \mathrm{L}$ was significantly lower than that of non-HDL-C $<2.11 \mathrm{mmol} / \mathrm{L}$ at 2 or $4 \mathrm{~h}$ (Figure 2D).

\section{DISCUSSION}

In this study, we found that when LDL-C goal $<1.4 \mathrm{mmol} / \mathrm{L}$ was used for evaluating cholesterol control in Chinese CHD patients after short-term statins treatment, the target percentage attainment in the non-fasting state was significantly higher than that of the fasting state. However, the percent attainment of non-fasting non-HDL-C was close to its fasting state, suggesting that non-HDL-C is more stable than LDL-C in assessing the percent attainment of non-fasting lipid for coronary heart disease patients. Notably, according to the new non-fasting cut-off points, $1.19 \mathrm{mmol} / \mathrm{L}$, the non-fasting goal attainment of LDL-C was close to its fasting value. 


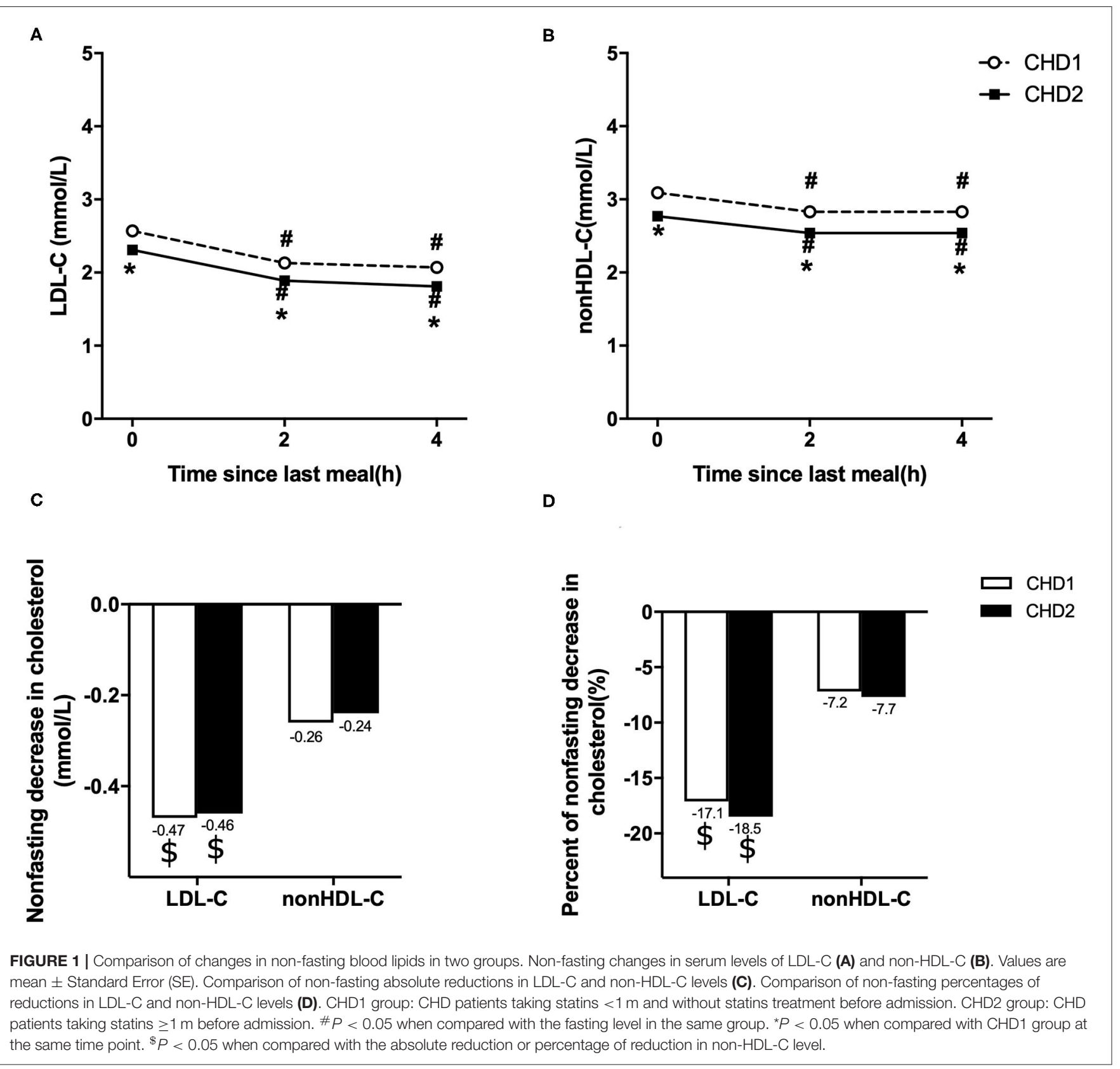

This suggests that lower non-fasting targets could be needed to evaluate the efficacy of cholesterol-lowing therapy in the non-fasting state, particularly when fasting blood lipids are unavailable and the percentage reduction of LDL-C cannot be determined due to a lack of baseline non-fasting levels before treatment.

There are two targets to evaluate the efficacy of cholesterollowering treatment in CHD patients. First, LDL-C should achieve a $\geq 50 \%$ reduction from baseline or a goal $<1.4 \mathrm{mmol} / \mathrm{L}$ according to the 2019 European guidelines (1). However, this recommendation refers only to cholesterol control in the fasting state. In this study, the goal attainment of LDL-C reduction $\geq 50 \%$ could not be evaluated because the baseline fasting or nonfasting LDL-C levels before treatment could not be obtained in most patients in the CHD2 group. Under these circumstances, a physician can only make clinical judgments based on LDL-C goal levels. A considerable number of CHD patients from other locations visit physicians but forget to remain in a fasting state. This is a common situation in the outpatient department of our hospital. As a result, physicians have to assess cholesterol control using non-fasting measurement of blood lipids. According to the joint consensus statement of European Atherosclerosis Society and European Federation of Clinical Chemistry and Laboratory Medicine (5), the non-fasting detection of blood lipids can 
TABLE 2 | Changes in levels of blood lipids and albumin after a daily breakfast in $89 \mathrm{CHD}$ patients.

\begin{tabular}{lccc}
\hline & Fasting & 2h after meal & 4 h after meal \\
\hline $\mathrm{TG}(\mathrm{mmol} / \mathrm{L}, \mathrm{SD})$ & $2.09 \pm 1.93$ & $2.34 \pm 1.88$ & $2.63 \pm 2.19$ \\
$\mathrm{TC}(\mathrm{mmol} / \mathrm{L}, \mathrm{SD})$ & $4.02 \pm 1.07$ & $3.72 \pm 0.96^{\star}$ & $3.71 \pm 0.96^{\star}$ \\
$\mathrm{HDL}-\mathrm{C}(\mathrm{mmol} / \mathrm{L}, \mathrm{SD})$ & $1.03 \pm 0.22$ & $1.01 \pm 0.21^{\star}$ & $1.03 \pm 0.28^{\star}$ \\
LDL-C $(\mathrm{mmol} / \mathrm{L}, \mathrm{SD})$ & $2.51 \pm 0.95$ & $2.11 \pm 0.71^{\star}$ & $2.07 \pm 0.74^{\star}$ \\
Non-HDL-C (mmol/L, SD) & $2.99 \pm 1.10$ & $2.71 \pm 0.89^{\star}$ & $2.69 \pm 0.97^{\star}$ \\
Albumin $(\mathrm{g} / \mathrm{L}, \mathrm{SD})$ & $38.3 \pm 2.85$ & $38.3 \pm 3.07$ & $38.5 \pm 3.00$ \\
\hline
\end{tabular}

TG, triglyceride; TC, total cholesterol; HDL-C, high-density lipoprotein cholesterol; LDL-C, low-density lipoprotein. CHD patients including patients in CHD1 group and CHD2 group. Data are mean $\pm S D$. ${ }^{*} P<0.05$ when compared with fasting state.

be routinely applied in $\mathrm{CHD}$ patients as long as they are willing to undergo non-fasting measurement. This suggests that measurement of LDL-C level in the non-fasting state is quite important.

Compared with some studies with large population in other countries $(10,11,17,18)$, the reduction in LDL-C level in CHD patients after a daily meal was more significant in the present study. The maximum mean reduction in LDL-C or non-HDL$\mathrm{C}$ was $\sim 0.1-0.2 \mathrm{mmol} / \mathrm{L}$ in the European and North American subjects $(10,11,17,18)$; however, Chinese CHD patients in this study showed a greater decrease in either directly detected LDLC ( i.e., 0.4-0.5 mmol/L) or calculated non-HDL-C (i.e., 0.2$0.3 \mathrm{mmol} / \mathrm{L}$ ) after a daily breakfast. Although our recent study showed that the post-prandial decline (i.e., $0.3-0.4 \mathrm{mmol} / \mathrm{L}$ ) in calculated LDL-C was less than that of the directly detected LDL at 2-4 h after a daily breakfast in Chinese CHD patients (9), it was still more than the reduction of above the European and North American studies $(10,11,17,18)$. The underling mechanisms of non-fasting reduction in LDL-C may be complicated in the present study. First, in the Copenhagen General Population Study, they compared blood lipids levels of individuals at random time points after the last meal in the large-scale population. By contrast, our measurements were acquired from the same individuals at various times since the last meal, which was different from the Copenhagen General Population Study in terms of the observation time-points and monitoring method. Second, post-prandial reduction in LDL-C concentration is most likely haemodilution resulting from fluid intake in relation to the meal and thus adjusting the data for albumin concentration was recommended $(7,10)$. Langsted et al. (10) observed that the nonfasting LDL-C concentration no longer changed after adjustment for albumin concentration. However, a very slight change in the post-prandial albumin level was observed in our study; therefore, haemodilution may not be the only cause of post-prandial decline in the LDL-C level in the Chinese. Third, the diet structures of Chinese and western people are very different. For example, the Chinese people prefer carbohydrates (16). It is not clear whether the high-carbohydrate diet will cause a more significant decline in cholesterol. At any rate, the obvious decrease in non-fasting LDL-C might affect the evaluation of goal attainment when the LDL-C level was detected after a meal.
Indeed, non-HDL-C was more stable than LDL-C in assessing the percent attainment of non-fasting lipid for coronary heart disease patients. Non-fasting reduction in non-HDL-C was less than that in LDL-C and the difference between fasting and nonfasting percentage attainments of non-HDL-C $<2.2 \mathrm{mmol} / \mathrm{L}$ was less than that of LDL-C $<1.4 \mathrm{mmol} / \mathrm{L}$. Non-HDL-C represents the cholesterol content of all atherosclerotic lipoproteins in the circulation, including chylomicrons, very-low-density-lipid and their remnants, intermediate-density lipoproteins, LDL, and lipoprotein (a) particles. Takahiro found that non-HDL cholesterol levels were clearly associated with future mortality and were less affected by fasting status or serum triglyceride levels (27). Meta-analyses and prospective studies with large populations supported the opinion that on-treatment levels of non-HDL-C were stronger than that of LDL-C for future CVD risk estimation $(20,21)$. Furthermore, non-HDL-C is a cheaper equivalent predictor of risk on and off statins, without the requirement for a fasting sample (28). Therefore, some scholars proposed that the clinical benefit obtained from controlling nonHDL-C would be greater than the one obtained from controlling LDL-C (19-21, 23).

Nevertheless, the percent attainment of non-HDL-C was higher than that of LDL-C in both fasting and non-fasting states according to the goals of LDL-C $<1.4 \mathrm{mmol} / \mathrm{L}$ and non-HDL-C $<2.2 \mathrm{mmol} / \mathrm{L}$, respectively, in this study. The difference between non-HDL-C and LDL-C will increase with TG elevation, which could exert a substantial influence on evaluation of cholesterollowering treatment $(29,30)$. The fixed difference between fasting non-HDL-C and LDL-C goals was $30 \mathrm{mg} / \mathrm{dl}$ (i.e., $0.8 \mathrm{mmol} / \mathrm{L}$ ) when fasting TG level was $1.7 \mathrm{mmol} / \mathrm{L}$, reflecting the fact that cholesterol content within TG-rich lipoproteins was about 1.7/2.2 $\approx 0.8 \mathrm{mmol} / \mathrm{L}$. Some scholars found that the goal attainment of non-HDL-C was higher than that of LDL-C when fasting TG was $<1.7 \mathrm{mmol} / \mathrm{L}$, while it was less than that of LDL-C when fasting TG $>2.3 \mathrm{mmol} / \mathrm{L}$ (29). Su et al. reported that the specific and fixed goals as non-HDL-C $0.8 \mathrm{mmol} / \mathrm{L}(30 \mathrm{mg} / \mathrm{dL})$ higher than the corresponding LDL-C goals were not sufficient for Chinese patients with $\mathrm{CHD}$ and proposed that flexible goals basing on TG level were more appropriate (30). This is consistent with our findings that the percent attainment of LDL-C $<1.4 \mathrm{mmol} / \mathrm{L}$ was significantly lower than that of non-HDL-C $<2.2 \mathrm{mmol} / \mathrm{L}$ in the fasting state; however, the difference in percent attainment between LDL-C and non-HDL-C after a daily breakfast became smaller with the increase in non-fasting TG level.

It was found that the percent attainment of post-prandial LDL-C was significantly higher than that of fasting values in the present study, suggesting that the fasting goals of LDL-C $<1.4$ $\mathrm{mmol} / \mathrm{L}$ was indeed unsuitable for the evaluation of post-prandial cholesterol control. ROC analysis has been used to identify the optimal cut-off point for the diagnosis of post-prandial hypertriglyceridemia $(16,31,32)$ but not for determining goals of LDL-C and non-HDL-C in the non-fasting state corresponding to the fasting goals. Because the non-fasting cut-off points acquired by ROC analysis corresponded to the fasting goals of LDL-C $<1.4 \mathrm{mmol} / \mathrm{L}$ and non-HDL-C $<2.2 \mathrm{mmol} / \mathrm{L}$, the postprandial percent attainments were very similar to their respective fasting values. This suggested that lower post-prandial cut-off 
A

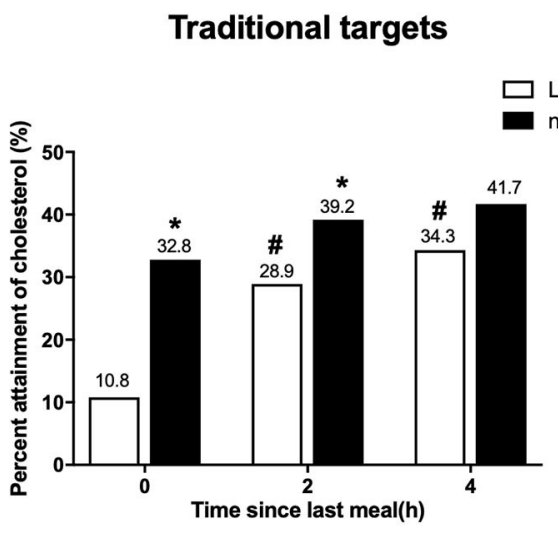

C

ROC curve estimated for pnonHDL-C $4 h$

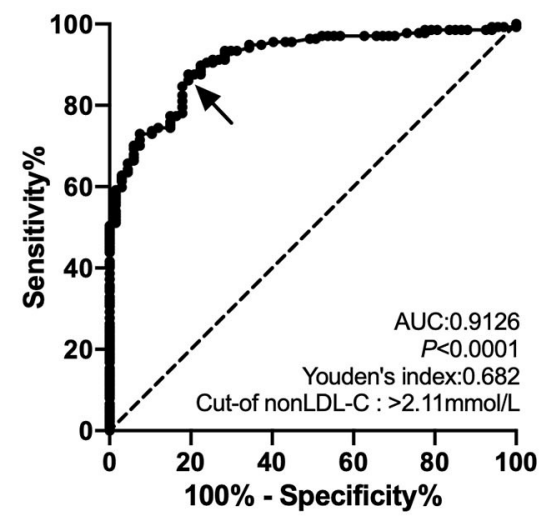

B

\section{ROC curve estimated for pLDL-C $4 \mathrm{~h}$}

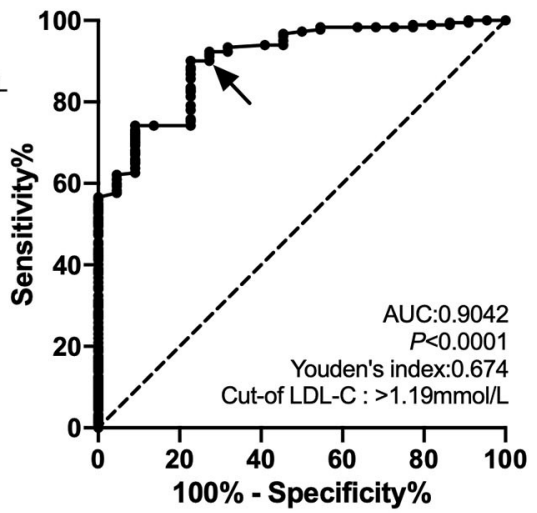

D

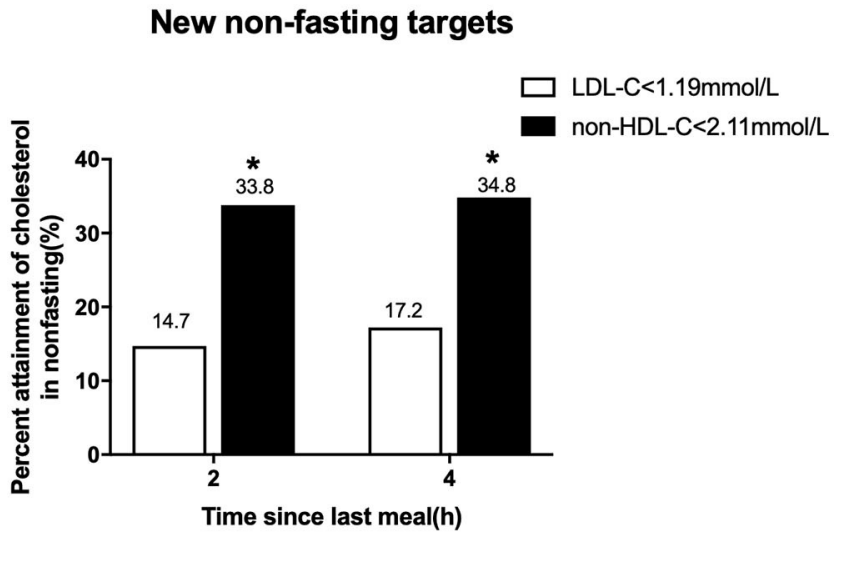

FIGURE 2 | Comparison of the percent attainments of LDL-C and non-HDL-C levels according to different targets in fasting and non-fasting states in Group CHD2. CHD2 group: CHD patients taking statins $\geq 1 \mathrm{~m}$ before admission. (A) Comparison of the percent attainments in both fasting and non-fasting states according to the recommended targets of LDL-C level $<1.4 \mathrm{mmol} / \mathrm{L}$ and non-HDL-C level $<2.2 \mathrm{mmol} / \mathrm{L}$. (B,C) Non-fasting cut-off points in relation to fasting $L D L-C$ level $1.4 \mathrm{mmol} / \mathrm{L}$ (B) and non-HDL-C level $2.2 \mathrm{mmol} / \mathrm{L}$ (C) determined by ROC analysis and Youden's index (marked by the solid arrows). (D) Comparison of the percent attainments according to non-fasting cut-off points of LDL-C $<1.19 \mathrm{mmol} / \mathrm{L}$ and non-HDL-C $<2.11 \mathrm{mmol} / \mathrm{L}$. $\# P<0.05$ when compared with the percent attainment of the same target(s) in the fasting state. ${ }^{*} P<0.05$ when compared with the percent attainment of different LDL-C target at the same time point.

points, different from their fasting goals, should be adopted in the evaluation of post-prandial goal attainment, unless it is possible to assess the percentage reduction in the non-fasting LDL-C level. In this study, the difference ( 1.19 vs. $2.11 \mathrm{mmol} / \mathrm{L})$ between non-fasting cut-off points of LDL-C and non-HDL-C was 0.92 $\mathrm{mmol} / \mathrm{L}$ corresponding to non-fasting TG level of $\sim 2.0 \mathrm{mmol} / \mathrm{L}$ (i.e., $0.92 \times 2.2=2.024 \approx 2.0$ ). This suggests that a larger difference between LDL-C and non-HDL-C should be considered in the evaluation of non-fasting goal attainment even after a daily meal without high fat.

This study had some limitations. First, it was a single center study with a small sample size of inpatients. In the future, the suitability of non-fasting cut-off points in a large sample of arteriosclerotic cardiovascular disease patients, including patients with ischemic stroke and peripheral vascular disease, is worth exploring. Second, only the percent attainment of the goal, but not percentage reduction of LDL-C, was evaluated because of the lack of baseline levels of blood lipids. Third, it can only reflect the situation of patients admitted to the hospital. There are also a large number of outpatients with relatively stable conditions and their medication situation may be different from that of hospitalized patients. Therefore, the changes of post-prandial blood lipid may be different from our study. This needs further study in the future.

\section{CONCLUSIONS}

Non-HDL-C is more stable than LDL-C in assessing the percent attainment of non-fasting lipid for coronary heart disease patients. If we want to use LDL-C to assess the percent attainment of post-prandial blood lipids, we may need to determine a lower non-fasting cut-off point. 


\section{DATA AVAILABILITY STATEMENT}

The original contributions generated for the study are included in the article/Supplementary Material, further inquiries can be directed to the corresponding author/s.

\section{ETHICS STATEMENT}

Written informed consent was obtained from the individual(s) for the publication of any potentially identifiable images or data included in this article.

\section{AUTHOR CONTRIBUTIONS}

L-LG, Y-qC, and LL designed and conducted of this study. Q-zL, FT, Q-YX, and L-yZ participated in the collection, analysis, and

\section{REFERENCES}

1. Mach F, Baigent C, Catapano AL, Koskinas KC, Casula M, Badimon L, et al. 2019 ESC/EAS Guidelines for the management of dyslipidaemias: lipid modification to reduce cardiovascular risk. Eur Heart J. (2019) 41:1118. doi: 10.15829/1560-4071-2020-3826

2. Pastromas S, Terzi AB, Tousoulis D, Koulouris S. Postprandial lipemia: an under-recognized atherogenic factor in patients with diabetes mellitus. Int $J$ Cardiol. (2008) 126:3-12. doi: 10.1016/j.ijcard.2007.04.172

3. Doran B, Guo Y, Xu J, Weintraub H, Mora S, Maron DJ, et al. Prognostic value of fasting versus nonfasting low-density lipoprotein cholesterol levels on long-term mortality: insight from the National Health and Nutrition Examination Survey III (NHANES-III). Circulation. (2014) 130:546-53. doi: 10.1161/CIRCULATIONAHA.114.010001

4. Langsted A, Nordestgaard BG. Nonfasting versus fasting lipid profile for cardiovascular risk prediction. Pathology. (2019) 51:131-41. doi: 10.1016/j.pathol.2018.09.062

5. Nordestgaard BG, Langsted A, Mora S, Kolovou G, Baum H, Bruckert E, et al. Fasting is not routinely required for determination of a lipid profile: clinical and laboratory implications including flagging at desirable concentration cutpoints-a joint consensus statement from the European Atherosclerosis Society and European Federation of Clinical Chemistry and Laboratory Medicine. Eur Heart J. (2016) 37:1944-58. doi: 10.1093/eurheartj/ehw152

6. Steen DL, Khan I, Ansell D, Sanchez RJ, Ray KK. Retrospective examination of lipid-lowing treatment patterns in a real-world high-risk cohort in the UK in 2014: comparison with the National Institute for Health and Care Excellence (NICE) 2014 lipid modification guidelines. BMJ Open. (2017) 7:e013255. doi: 10.1136/bmjopen-2016-013255

7. Langsted A, Nordestgaard BG. Nonfasting lipids, lipoproteins, and apolipoproteins in individuals with and without diabetes: 58434 individuals from the Copenhagen General Population Study. Clin Chem. (2011) 57:482-9. doi: 10.1373/clinchem.2010.157164

8. Downs JR, O'Malley PG. Management of dyslipidemia for cardiovascular disease risk reduction: synopsis of the 2014. U.S. Department of Veterans Affairs and U.S. Department of Defense clinical practice guideline. Ann Intern Med. (2015) 163:291-7. doi: 10.7326/M15-0840

9. Lin QZ, Chen YQ, Guo LL, Xiang QY, Tian F, Wen T, et al. Comparison of non-fasting LDL-C levels calculated by Friedewald formula with those directly measured in Chinese patients with coronary heart disease after a daily breakfast. Clin Chim Acta. (2019) 495:399-405. doi: 10.1016/j.cca.2019.05.010

10. Langsted A, Freiberg JJ, Nordestgaard BG. Fasting and nonfasting lipid levels: influence of normal food intake on lipids, lipoproteins, apolipoproteins, and cardiovascular risk prediction. Circulation. (2008) 118:2047-56. doi: 10.1161/CIRCULATIONAHA.108.804146 interpretation of the data. L-LG and TW contributed to the preparation of the manuscript. LL carried out the approval of the study. All authors read the study and approved the manuscript for publication.

\section{FUNDING}

This study has received support from the National Natural Science Foundation of China (grant numbers 81270956 and 81470577).

\section{SUPPLEMENTARY MATERIAL}

The Supplementary Material for this article can be found online at: https://www.frontiersin.org/articles/10.3389/fcvm. 2021.649181/full\#supplementary-material

11. Mora S, Rifai N, Buring JE, Ridker PM. Fasting compared with nonfasting lipids and apolipoproteins for predicting incident cardiovascular events. Circulation. (2008) 118:9931001. doi: 10.1161/CIRCULATIONAHA.108.777334

12. Stone NJ, Robinson JG, Lichtenstein AH, Bairey Merz CN, Blum CB, Eckel RH, et al. 2013 ACC/AHA guideline on the treatment of blood cholesterol to reduce atherosclerotic cardiovascular risk in adults: a report of the American College of Cardiology/American Heart Association Task Force on Practice Guidelines. J Am Coll Cardiol. (2014). 63:2889934. doi: 10.1161/01.cir.0000437738.63853.7a

13. Joint committee for guideline revision National Expert Committee on Cardiovascular Diseases, National Center for Cardiovascular Diseases Chinese Society of Cardiology, Chinese Medical Association Chinese Diabetes Society, Chinese Medical Association Chinese Society of Endocrinology, Chinese Medical Association Chinese Society of laboratory Medicine, Chinese Medical Association Writing Group Members Group Leader, et al. 2016 Chinese guidelines for the management of dyslipidemia in adults. J Geriatr Cardiol. (2018). 15:1-29. doi: 10.11909/j.issn.1671-5411.2018.01.011

14. Catapano AL, Graham I, De Backer G, Wiklund O, Chapman MJ, Drexel H, et al. 2016 ESC/EAS guidelines for the management of dyslipidaemias. Eur Heart J. (2016) 37:2999-3058. doi: 10.1093/eurheartj/ehw272

15. Grundy SM, Stone NJ, Bailey AL, Beam C, Birtcher KK, Blumenthal RS, et al. 2018 AHA/ACC/AACVPR/AAPA/ABC/ACPM/ ADA/AGS/APhA/ASPC/NLA/PCNA Guideline on the Management of Blood Cholesterol: A Report of the American College of Cardiology/American Heart Association Task Force on Clinical Practice Guidelines. J Am Coll Cardiol. (2019). 73:e285-350. doi: 10.1016/j.jacc.2018.11.004

16. Tian F, Xiang QY, Zhang MY, Chen YQ, Lin QZ, Wen T, et al. Changes in non-fasting concentrations of blood lipids after a daily Chinese breakfast in overweight subjects without fasting hypertriglyceridemia. Clin Chim Acta. (2019) 490:147-53. doi: 10.1016/j.cca.2019.01.004

17. Sidhu D, Naugler C. Fasting time and lipid levels in a community-based population: a cross-sectional study. Arch Intern Med. (2012) 172:170710. doi: 10.1001/archinternmed.2012.3708

18. Bansal S, Buring JE, Rifai N, Mora S, Sacks FM, Ridker PM. Fasting compared with nonfasting triglycerides and risk of cardiovascular events in women. JAMA. (2007) 298:309-16. doi: 10.1001/jama.298.3.309

19. Benn M. Apolipoprotein B levels, APOB alleles, and risk of ischemic cardiovascular disease in the general population, a review. Atherosclerosis. (2009) 206:17-30. doi: 10.1016/j.atherosclerosis.2009.01.004

20. Toth PP. Association of LDL cholesterol, non-HDL cholesterol, and apolipoprotein B levels with risk of cardiovascular events among patients treated with statins: a meta-analysis. Yearbook Endocrinol. (2012) 2012:658. doi: 10.1016/j.yend.2012.05.028 
21. Ridker PM, Rifai N, Cook NR, Bradwin G, Buring JE. Non-HDL cholesterol, apolipoproteins A-I and B100, standard lipid measures, lipid ratios, and CRP as risk factors for cardiovascular disease in women. JAMA. (2005) 294:326-33. doi: 10.1001/jama.294.3.326

22. Di Angelantonio E, Gao P, Pennells L, Kaptoge S, Caslake M, Thompson A, et al. Lipid-related markers and cardiovascular disease prediction. JAMA. (2012) 307:2499-506. doi: 10.1001/jama.2012.6571

23. Carbayo Herencia JA, Simarro Rueda M, Palazon Bru A, Molina Escribano F, Ponce Garcia I, Artigao Rodenas LM, et al. Evaluation of nonHDL cholesterol as a predictor of non-fatal cardiovascular events in a prospective population cohort. Clin Investig Arterioscler. (2018) 30:6471. doi: 10.1016/j.artere.2017.10.003

24. de Vries M, Klop B, Castro Cabezas M. The use of the non-fasting lipid profile for lipid-lowering therapy in clinical practice - point of view. Atherosclerosis. (2014) 234:473-5. doi: 10.1016/j.atherosclerosis.2014. 03.024

25. Zhao Y, Peng R, Zhao W, Liu Q, Guo Y, Zhao S, et al. Zhibitai and low-dose atorvastatin reduce blood lipids and inflammation in patients with coronary artery disease. Medicine. 96:e6104. doi: 10.1097/MD.0000000000006104

26. Tian F, Wu CL, Yu BL, Liu L, Hu JR. Apolipoprotein O expression in mouse liver enhances hepatic lipid accumulation by impairing mitochondrial function. Biochem Biophys Res Commun. (2017) 491:8-14. doi: 10.1016/j.bbrc.2017.06.128

27. Ito $\mathrm{T}$, Arima H, Fujiyoshi A, Miura K, Takashima N, Ohkubo T, et al. Relationship between non-high-density lipoprotein cholesterol and the longterm mortality of cardiovascular diseases: NIPPON DATA 90. Int J Cardiol. (2016) 220:262-7. doi: 10.1016/j.ijcard.2016.06.021

28. Welsh C, Celis-Morales CA, Brown R, Mackay DF, Lewsey J, Mark PB, et al. Comparison of conventional lipoprotein tests and apolipoproteins in the prediction of cardiovascular disease. Circulation. (2019) 140:54252. doi: 10.1161/CIRCULATIONAHA.119.041149

29. Al-Hashmi K, Al-Zakwani I, Al Mahmeed W, Arafah M, Al-Hinai AT, Shehab A, et al. Non-high-density lipoprotein cholesterol target achievement in patients on lipid-lowering drugs and stratified by triglyceride levels in the Arabian Gulf. J Clin Lipidol. (2016) 10:368-77. doi: 10.1016/j.jacl.2015.12.021

30. Su X, Luo M, Tang X, Luo Y, Zheng X, Peng D, et al. Goals of non-high density lipoprotein cholesterol need to be adjusted in Chinese acute coronary syndrome patients: findings from the CCC-ACS project. Clin Chim Acta. (2019) 496:48-54. doi: 10.1016/j.cca.2019.06.022

31. White KT, Moorthy MV, Akinkuolie AO, Demler O, Ridker PM, Cook NR, et al. Identifying an optimal cutpoint for the diagnosis of hypertriglyceridemia in the nonfasting state. Clin Chem. (2015) 61:115663. doi: $10.1373 /$ clinchem.2015.241752

32. Sevilla-Gonzalez MDR, Aguilar-Salinas CA, Munoz-Hernandez L, AlmedaValdes P, Mehta R, Zubiran R, et al. Identification of a threshold to discriminate fasting hypertriglyceridemia with postprandial values. Lipids Health Dis. (2018) 17:156. doi: 10.1186/s12944-018-0803-8

Conflict of Interest: The authors declare that the research was conducted in the absence of any commercial or financial relationships that could be construed as a potential conflict of interest.

Copyright (C) 2021 Guo, Chen, Lin, Tian, Xiang, Zhu, Xu, Wen and Liu. This is an open-access article distributed under the terms of the Creative Commons Attribution License (CC BY). The use, distribution or reproduction in other forums is permitted, provided the original author(s) and the copyright owner(s) are credited and that the original publication in this journal is cited, in accordance with accepted academic practice. No use, distribution or reproduction is permitted which does not comply with these terms. 